\title{
Comparison of technologies for deorbiting spacecraft from low-earth-orbit at end of mission
}

\author{
G. Sánchez-Arriaga $^{\text {a, }}{ }^{*}$, J.R. Sanmartín ${ }^{\text {b }}$, E.C. Lorenzini ${ }^{\text {c, } 1}$

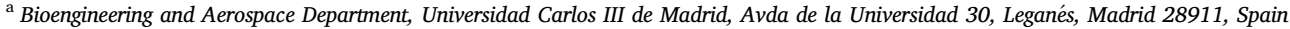 \\ ${ }^{\mathrm{b}}$ Departamento de Física Aplicada a la Ingeniería Aeronáutica y Naval, Universidad Politécnica de Madrid, Madrid 28040, Spain \\ ${ }^{c}$ Department of Industrial Engineering, University of Padova, Padova, Italy
}

\section{A B S T R A C T}

An analytical comparison of four technologies for deorbiting spacecraft from Low-Earth-Orbit at end of mission is presented. Basic formulas based on simple physical models of key figures of merit for each device are found. Active devices - rockets and electrical thrusters - and passive technologies - drag augmentation devices and electrodynamic tethers - are considered. A basic figure of merit is the deorbit device-to-spacecraft mass ratio, which is, in general, a function of environmental variables, technology development parameters and deorbit time. For typical state-of-the-art values, equal deorbit time, middle inclination and initial altitude of $850 \mathrm{~km}$, the analysis indicates that tethers are about one and two orders of magnitude lighter than active technologies and drag augmentation devices, respectively; a tether needs a few percent mass-ratio for a deorbit time of a couple of weeks. For high inclination, the performance drop of the tether system is moderate: mass ratio and deorbit time increase by factors of 2 and 4, respectively. Besides collision risk with other spacecraft and system mass considerations, such as main driving factors for deorbit space technologies, the analysis addresses other important constraints, like deorbit time, system scalability, manoeuver capability, reliability, simplicity, attitude control requirement, and re-entry and multi-mission capability (deorbit and re-boost) issues. The requirements and constraints are used to make a critical assessment of the four technologies as functions of spacecraft mass and initial orbit (altitude and inclination). Emphasis is placed on electrodynamic tethers, including the latest advances attained in the FP7/Space project BETs. The superiority of tape tethers as compared to round and multi-line tethers in terms of deorbit mission performance is highlighted, as well as the importance of an optimal geometry selection, i.e. tape length, width, and thickness, as function of spacecraft mass and initial orbit. Tether system configuration, deployment and dynamical issues, including a simple passive way to mitigate the well-known dynamical instability of electrodynamic tethers, are also discussed.

\section{Introduction}

In 1978, NASA scientists D. J. Kessler and B. G. Cour-Palais predicted that, around the year 2000, the population of catalogued debris would be so dense that collisional breakup of satellites would become a new source for additional satellite debris and, in certain regions near the Earth, may quickly exceed the natural meteoroid flux [1]. The large amount of objects produced at each collision over a longer period of time, even though a zero net input rate may be maintained, would produce an exponential growth in the collision rate. In this pioneering work, it was suggested that reducing the projected number of large, nonoperational satellites and improving engineering designs to avoid satellite break ups from structural failure and explosion in space were effective methods to modify this catastrophic trend.

Although important progresses were achieved, like the one related with spacecraft passivation $[2,3]$ and also the important IADC guideline to ensure that the lifetime after disposal will not exceed 25 years, measures adopted by space agencies and industry now seem to be insufficient to stabilize the debris population. Even with a $90 \%$ compliance of the guidelines, Low Earth Orbit (LEO) population will increase about 30\% over the next 200 years and catastrophic collision, i.e. total break-up of the target, will occur every 5-9 years [4]. The continuous growth of launches during the last decades, and some events, like the anti-satellite missile test in 2007 and the collision between the communication satellites Iridium 33 and Cosmos 2251 in 2009, degraded the space debris environment. LEO with altitudes between $800-1000 \mathrm{~km}$ and inclinations at $71^{\circ}, 82^{\circ}$ and $98^{\circ}$ are the most critical. Two actions may be essential to stabilize the space debris population. First, spacecrafts should be equipped with a deorbit kit that will produce the re-entry at the end-of-life. Second, Active Debris Removal (ADR) missions to capture the most dangerous objects and force their deorbiting are required in highly populated orbits. Both actions need an efficient deorbit technology and 


\begin{tabular}{|llll|}
\hline \multicolumn{2}{|c|}{ Nomenclature } & $m_{p}$ & Propellant mass \\
$\boldsymbol{B}$ & Magnetic field & $m_{r}$ & Rocket mass \\
$\boldsymbol{E}_{m}$ & Motional electric field & $m_{s}$ & Spacecraft mass \\
$h$ & Tether thickness & $m_{t}$ & Tether system mass \\
$H$ & Spacecraft altitude & $N_{c}$ & Number of fatal impact \\
$H_{O}$ & Initial Altitude & $R_{E}$ & Earth Radius \\
$H_{F}$ & Final Altitude & $\boldsymbol{v}$ & Spacecraft velocity \\
$i$ & Orbit inclination & $w$ & Tether width \\
$L$ & Tether Length & $\ddot{\mathrm{E}}$ & Earth gravitational parameter \\
$\mathrm{m}_{c}$ & Conductive tether mass & $\rho_{O}$ & Air density \\
$m_{D A D}$ & Drag augmentation device mass & $\rho_{t}$ & Tether density \\
$m_{e}$ & Electric propulsion system mass & $\sigma$ & Tether conductivity \\
\hline
\end{tabular}

ADR also needs a capture technology like a robotic arms, nets and harpoons. Once the space debris population falls below the Kessler critical density, ADR missions will not be implemented, and the space will be kept clean by equipping the spacecraft with its own deorbit kit. It is expected that, linked to the deterioration of the space environment and the probable appearance of a tougher legal framework, a new market on deorbit technologies will open in the next future.

Deorbit technologies can be classified into two categories. Active technologies, including chemical and electric propulsion, expel a propellant at high velocity and in the correct direction to deorbit the spacecraft. Passive technologies, involving air drag augmentation devices (DADs) and bare electrodynamic tethers (BET), are based on air drag and magnetic drag. Active technologies reached a higher level of maturity whereas the development of passive devices has been revitalized during the last decades mostly driven by the space debris problem. The absence of mature devices and the existence of a long flight history for some of them make it difficult to identify the deorbit technology that will dominate the market in the next decades. However, the selection of the most competitive technology may be based on a set of desirable properties or requirements that can be anticipated. The following list was presented at the 51th Session of COPUOS Scientific and Technical Subcommittee [5]:

1. Bring deorbit time below some threshold (25 years maximum for initial orbit at critical altitudes and inclinations).

2. Be a small mass fraction of its spacecraft.

3. Allow scalable design for a wide range of spacecraft mass and reaching into multi-ton mass range.

4. Allow maneuvers in case of long deorbiting to avoid large trackable debris.

5. Be simple and reliable. In particular, be ready to start operating with minimum support after lying dormant for years.

Somehow related with requirement (1), the technology should

6. Decrease the frontal area by deorbit time product, $A \times t_{D}$, or demonstrate that, in case of collision, it will not damage other operative spacecraft.

Requirements (1)-(6) seem to be essential. Two additional requirements that would make a particular technology even more attractive are

7. Allow controlled re-entry.

8. Be able to produce spacecraft deorbiting and reboost in multi-mission scenarios like for instance in ADR missions where several objects should be captured and deorbited.

At a first sight, condition (7) may appear as essential to avoid onground damage by space debris fragments. However, spacecraft with mass well below $1 \mathrm{t}$ are fully burned during the re-entry, and their probability of damage on ground is small. High mass objects will not be troublesome if the Design for Demise solution is implemented.

This work will make an analytical comparison of the four deorbit technologies according to criteria (1)-(8), the state-of-the-art and, for some cases, progresses that are expected. Section 2 shows simple analytical models of the deorbit devices performance as a function of technological parameter, environmental variables and design considerations. Based on these models, the state-of-the art of the different devices and the experiences learned in past mission, the technologies are compared according to criteria (1)-(8) in Section 3. Specific considerations applied to electrodynamics tethers are discussed in Section 4. Section 5 summarizes the conclusions of the work.

\section{Deorbit technologies performance}

We consider a spacecraft of mass $\mathrm{m}_{\mathrm{s}}$ orbiting in a circular orbit of altitude $\mathrm{H}_{0}$. If equipped with a deorbit technology that produces a strong thrust in a short time, then the most efficient deorbiting manoeuver is a Hohmann transfer. The total velocity impulse, $\Delta v$, required for a twoburn transfer between circular orbits at altitudes $\mathrm{H}_{0}$ and $\mathrm{H}_{\mathrm{F}}$ is

$\Delta v=\frac{1}{2} \sqrt{\frac{\mu_{E}}{R_{E}+H_{0}}} \frac{H_{0}-H_{F}}{R_{E}+H_{0}}$

where $\ddot{\mathrm{E}}_{\mathrm{E}}$ and $\mathrm{R}_{\mathrm{E}}$ are the Earth Gravitational parameter and radius. By contrast, other deorbit technologies produce low thrusts during long times, thus yielding a spiral transfer. For these cases, the scalar product of the equation of motion of the spacecraft by the orbital velocity $\mathbf{v}$ yields

$\frac{d H}{d t}=\frac{2\left(R_{E}+H\right)^{2}}{\mu} \frac{\boldsymbol{F}_{p} \cdot \boldsymbol{v}}{m_{s}}$

where we used the relation $v \approx \sqrt{\mu_{E} / R_{E}+H}$ valid for spiral transfer and $\mathbf{F}_{\mathrm{p}}$ is the perturbation force produced by the deorbit device. As shown below, Eqs. (1) and (2) can be used to find the performance of all the deorbit technologies of interest.

\subsection{Chemical propulsion}

Due to reliability and simplicity requirements, solid-propellant rockets are recommended for deorbiting. Using Tsiolkovsky rocket equation, $\Delta v=c_{e x} \ln \left[1+m_{p} /\left(m_{s}+k_{r} m_{p}\right)\right]$, where $\Delta v$ is taken from Eq. (1), yields

$\frac{m_{r}}{m_{s}}=\left(1+k_{r}\right)\left(\frac{1}{e^{\Delta v / c_{e x}}-1}-k_{r}\right)^{-1}$

where $m_{p}$ is the mass of propellant, $m_{r}=\left(1+k_{r}\right) m_{p}$ is the mass of the rocket system, $c_{e x}$ is the effective exhaust velocity, and $k_{r}$ is the structuralto-propellant mass ratio. Typical values are $c_{e x}=2.6 \mathrm{~km} / \mathrm{s}$ and $k_{r}=0.25$, in the range between Shuttle boosters $\left(k_{r}=0.16\right)$ and the Star 12 engine $\left(k_{r}=0.5\right)$. In SPADES, a recent study by the CDF [6], small $\left(\mathrm{m}_{\mathrm{s}}<200 \mathrm{~kg}\right)$, 
medium $\left(1000<\mathrm{m}_{\mathrm{s}}<2000 \mathrm{~kg}\right)$ and large $\left(\mathrm{m}_{\mathrm{s}}>2000 \mathrm{~kg}\right)$ satellite $\mathrm{s}_{\mathrm{s}}$ were considered. The authors found the mass ratios $7 \%, 16.5 \%$ and $22 \%$, respectively, when using solid propellant. Mass ratio is halved by skipping the second Hohmann transfer if one lets the apogee decrease slowly under the action of the air drag over a large number of short passes at perigee (deorbit time would be of the order of years).

\subsection{Electric propulsion}

For a thrust $\boldsymbol{F}_{p}$ that is constant and always directed along the tangent to the orbit, one has $\boldsymbol{F}_{p} \cdot \boldsymbol{v}=-\dot{m} c_{e x} v$. When writing the propellant mass as $m_{p}=m t_{D}$, with $t_{\mathrm{D}}$ the deorbit time Eq. (2) gives

$\frac{m_{p}}{m_{s}}=\frac{1}{c_{e x}} \sqrt{\frac{\mu_{E}}{R_{E}+H_{0}}}\left(\sqrt{\frac{R_{E}+H_{0}}{R_{E}+H_{F}}}-1\right)$

where $m$ is the mass flow rate. The total mass of the electric propulsion system, $m_{e}=\left(1+k_{e}\right) m_{p}+m_{\text {power }}$, includes the propellant mass, the structural mass $\left(k_{e} m_{p}\right)$, and the mass of the power system ( $\left.\mathrm{m}_{\text {power }}\right)$. The total mass reaches the minimum $m_{e}=2\left(1+k_{e}\right) m_{p}$ for exhaust velocity equal to the Stuhlinger characteristic velocity, $c_{e x}=\sqrt{2 \eta\left(1+k_{e}\right) t_{D} / \alpha}$, where $\eta$ is the efficiency of the electric-to-flow energy conversion, and $\alpha$ is the inverse specific power. Substituting the Stuhlinger velocity in Eq. (4) yield

$\frac{m_{e}}{m_{s}}=\sqrt{\frac{2 \alpha\left(1+k_{e}\right)}{\eta t_{D}}} \sqrt{\frac{\mu_{E}}{R_{E}+H_{0}}}\left(\sqrt{\frac{R_{E}+H_{0}}{R_{E}+H_{F}}}-1\right)$

with the mass ratio decreasing as $1 / \sqrt{t_{D}}$. Typical values of state-of-theart electric thrusters have $k_{e}=0.12, \eta=0.65$ and $\alpha=20 \mathrm{~kg} / \mathrm{kW}$, which includes power processing unit, cable, gimbal, thermal control, structure and propellant feed.

\section{3. augmentation devices}

The power dissipated by the aerodynamic drag on a DAD, like a sail or a balloon, is $\boldsymbol{F}_{p} \cdot \boldsymbol{v}=-C_{D} \rho_{0} A_{D A D} v^{3} / 2$. It depends on the drag coefficient $C_{D}$, the air density $\rho_{0}$ and the frontal area of the device $A_{D A D}$, which is related with the mass of the DAD through the ballistic coefficient $b$ by $m_{D A D}=b A_{D A D}$. The air drag acting on the spacecraft is neglected, as we already did for the other technologies. The substitution of these relations in Eq. (2) provide the following mass ratio

$\frac{m_{D A D}}{m_{s}}=\frac{b}{C_{D} t_{D} \sqrt{\mu_{E}}} \int_{H_{F}}^{H_{0}} \frac{d H}{\rho_{0} \sqrt{R_{E}+H}}$

that decays as the inverse of $t_{D}$. Typical values are $C_{D}=2$ and $b=75 \mathrm{gr} / \mathrm{m}^{2}$ (including the sail or the balloon, booms, control mechanism, and additional auxiliary devices). This value is between DLR/ESA sail $\left(b=92 \mathrm{gr} / \mathrm{m}^{2}\right)$ and Cosmos $1\left(\mathrm{~b}=67 \mathrm{gr} / \mathrm{m}^{2}\right)$ [7]. Later calculations will use CIRA-2012 model under average solar activity to compute the air density profile $\rho_{0}(H)$ in Eq. (6).

\subsection{Electrodynamic tethers}

The power dissipated by the Lorentz drag upon a tape-like BET of length, width, thickness, conductivity and density given by $L, w, h, \sigma$ and $\rho_{\mathrm{t}}$, is

$\boldsymbol{F}_{p} \cdot \boldsymbol{v}=\boldsymbol{v} \cdot \int_{0}^{L} I(s) \boldsymbol{u}_{t} \times \boldsymbol{B} d s=-m_{c} \frac{\sigma}{\rho_{t}} E_{m}^{2} i_{a v}$

with $\mathbf{u}_{\mathrm{t}}$ as a tangent unit vector along the straight tether pointing in the direction of the electric current $\boldsymbol{I}=I(\boldsymbol{s}) \boldsymbol{u}_{t}, E_{m}=\boldsymbol{u}_{t} \cdot(\boldsymbol{v} \times \boldsymbol{B})$ as the motional electric field, B as the geomagnetic field, $m_{c}=\rho_{t} L w h$ as the mass of the tape conductive tether, and $i_{a v}$ as the dimensionless averaged current along the tether [8]. In Eq. (7) we neglected the aerodynamic drag upon the spacecraft, as we did for the other technologies. We also ignored the air drag acting upon the tether because, as shown in Section 3, it is about two orders of magnitude smaller than the Lorentz drag.

The dominant components of the BET system mass are the conductive tether, the Hollow-Cathode (HC), the bottle of expellant and the deployment mechanism. Writing the mass of the full BET system as $m_{B E T}=k_{B E T} m_{c}$ and using Eq. (7) in Eq. (2) yield

$\frac{m_{B E T}}{m_{s}}=\frac{\mu_{E} \rho_{t} k_{B E T}}{2 \sigma_{t} t_{D}} \int_{H_{F}}^{H_{0}} \frac{d H}{\left(R_{E}+H\right)^{2} i_{a v} E_{m}^{2}}$

Like a DAD, the mass ratio for BET also decays as the inverse $t_{D}$. Characteristic values for tether density and conductivity are $\rho_{t}=2700 \mathrm{~kg} / \mathrm{m}^{3}$ and $\sigma_{t}=3.54 \times 10^{7} \Omega^{-1} \mathrm{~m}^{-1}$. The factor $\mathrm{k}_{\mathrm{BET}}$ will depend on the technological development of a BET deorbit kit. In the FP7/ Space project BETs hardware components, including a proto type deployment mechanism, tape tether, power control unit, and HC were manufactured and tested. From these results, one may expect that a $k_{B E T}$ value between 2 and 4 will be achieved after integration of all the devices in a single system. A typical value of the motional electric field, $E_{m}=v B \cos i$, in a medium-inclination orbit is about $E_{m}=7.6 \mathrm{~km} / \mathrm{s} \times 0.4 G \cos (\pi / 4) \approx 160 \mathrm{~V} / \mathrm{km}$. The precise value of $i_{a v}$ depends on tether dimensions $\left(L / h^{2 / 3}\right)$ and environmental variables like plasma density and geomagnetic field [8]. For performance estimation, a value of about 0.25 is representative of a wide range of mission (more detailed calculation are presented in Section 4).

\section{Deorbit technology comparison}

The models introduced in Section 2 can be used to compare the performance of the four deorbit technologies according to criteria (1) and (2). Fig. 1 shows the BET-to-other system mass ratios versus deorbit time, obtained after dividing Eqs. (3), (5) and (6) by Eq. (8). The equations were evaluated using the characteristic values presented in Section 2. Initial and final altitudes were $\mathrm{H}_{0}=850 \mathrm{~km}$ and $\mathrm{H}_{\mathrm{F}}=300 \mathrm{~km}$, respectively. The analysis indicates that BETs are the lightest technology. Tethers are almost two orders of magnitudes lighter than DADs. Clearly, Lorentz drag dominates air drag at orbit altitudes where space debris is troublesome. This property is mainly due to the strength of the plasma density as well as the geomagnetic field and the very low air density in the region of interest. The Earth and Jupiter are probably the most advantageous planets for BET in the Solar System. As regards active technologies, the BET is more than one order of magnitude and between a factor 2 and 9 lighter than chemical rockets and electric thrusters,

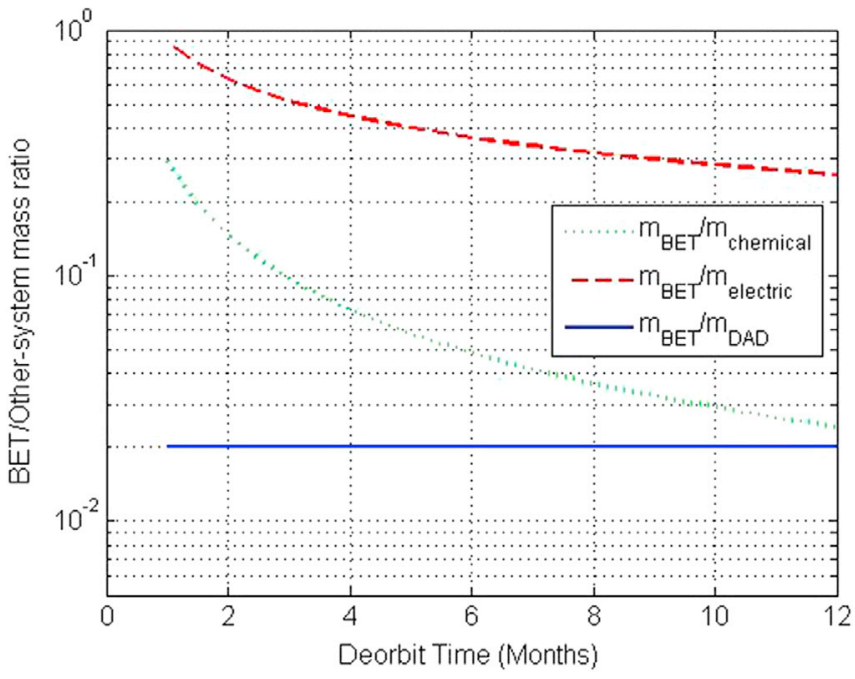

Fig. 1. BET-to-other technologies mass ratio versus deorbit time. 
respectively, for equal deorbit time. Since BET-to-spacecraft mass ratio decays as the inverse of $t_{D}$, BET becomes more competitive for longer missions. However, mission durations should be designed by taking into account possible tether cut by small debris [9]. The use of tape instead of round cross-sections is essential to achieve good BET performance with a very high survival probability [10] as specified later on.

A simple comparison between Lorentz drag and air-drag, at any LEO altitude, is given by their ratio [11],

$\frac{L B I_{a v}^{O M L}}{0.5 \quad A_{\text {sail }} C_{D} m_{i} N_{n} v^{2}} \sim \frac{A_{\text {front }}}{A_{\text {sail }}} \times \frac{N_{e}}{N_{n}} \times \frac{4 \sqrt{2}}{5 C_{D}} \sqrt{\frac{m_{i}}{m_{e}}}\left(\frac{L \Omega_{i}}{v}\right)^{3 / 2}$,

where a representative, moderate ohmic-effects, current law,

$I_{a v}^{O M L}=\frac{2}{5} e N_{e} \frac{L p}{\pi} \sqrt{\frac{2 e E_{m} L}{m_{e}}}, \quad\left(E_{m} \sim v B\right)$

was used, where $\Omega_{\mathrm{i}}$ is the ion gyrofrequency, $A_{\text {front }} \equiv L p / \pi$ is a representative tether frontal area ( $2 w L / \pi$ for a tape and $2 R L$ for a round tether) with $p$ the cross-section perimeter and $N_{e}$ and $N_{n}$ are the electron and neutral particle density, respectively. In $L E O$,

$\frac{v}{\Omega_{i}} \approx \frac{7.5 \mathrm{~km} / \mathrm{s}}{200 / \mathrm{s}} \approx 40 \quad \mathrm{~m}$

making for an extremely greater Lorentz drag in case of equal frontal areas, and equal plasma and neutral densities, and tether length $L$ in the kilometer range. The ratio given by Eq. (9) is typically between 2 and 2 orders of magnitude.

A similar comparison applies for Lorentz and Coulomb drag [12], just replacing $N_{n}$ with plasma density, $N_{i}=N_{e}$ itself, and writing

Coulomb drag $\sim \quad L r_{\max } \times m_{i} N_{i} v^{2}$

where $r_{\max }$ is a characteristic reach of the Coulomb field, basically dependent on the potential supplied to the tether by a power source, and both plasma density, and Debye length, which is typically a fraction of centimeter in $L E O$. Taking $r_{\text {max }}$ approximately equal to the sheath radius, $r_{\max } \sim r_{s h} \sim\left(\lambda_{D e} / 2\right) \sqrt{e V_{t} / k T_{e}}$ with $V_{t}$ the tether bias (see Ref. [13]) and writing the perimeter of the tape as twice its width, $p=2 w$, one finds the following ratio

$\frac{\text { Lorentz drag }}{\text { Coulomb drag }} \sim \frac{2 \sqrt{2}}{5 \pi} \times \frac{w}{\lambda_{D e}} \times \sqrt{\frac{k T_{e}}{e V_{t}}} \times \sqrt{\frac{m_{i}}{m_{e}}}\left(\frac{L \Omega_{i}}{v}\right)^{3 / 2} \sim 600$,

where we took $w \sim \lambda_{D e}, L=5 \mathrm{~km}$, Oxygen ions, $k T_{e}=0.2 \mathrm{eV}$ and $V_{t}=1 \mathrm{kV}$. Therefore Coulomb drag is very small against Lorentz drag, except for deorbiting very small satellites, requiring short tethers. Note also that the required power on-board to polarize the tether at such a high value can be also troublesome. Interestingly, the case of Coulomb drag versus Lorentz drag in the Solar wind is quite the opposite, because both $v / \Omega_{\mathrm{i}}$ and Debye length are very much larger than in $L E O$.

Criterion (3), scalable design reaching the multi-ton range, is an indispensable characteristic for practical deorbit technologies. If a given device could be easily adapted to be competitive within a spacecraft mass range from tens of kilograms to multiple tons, then it would immediately become advantageous due to cost considerations. Furthermore, large spacecrafts are the most dangerous because collision probability increases with frontal area and the number of fragments in case of collision with an object. Although with a high cost in term of mass (see Fig. 1), active technologies satisfy this criterion. BETs also satisfy it; an adequate selection of tether length, width and thickness, allows designing efficient and safe missions for critical mass and orbit altitudes and inclinations [9]. It is remarkable that different tape tether geometries with length, width and thickness within the ranges $L=1-5 \mathrm{~km}, w=0.5-2.5 \mathrm{~cm}$, and $h=50-100 \mu \mathrm{m}$, respectively, would not affect significantly to other BET systems like HC, deployment mechanism or power control unit. Recurrent cost would be low because the same hardware, probably with a few numbers of minor modifications, could be used. DADs may present difficulties with criterion (3) because multi-ton spacecrafts would need extremely large devices.

Chemical rockets are not affected by criterion (4) if two Hohmann transfers are carried out. The product of the mission duration by the cross-section area would be small, and a low collision probability would exist. However, maneuver capability is desirable for low impulse deorbit technologies, which may avoid large trackable debris during long deorbit missions. Spacecrafts equipped with electric propulsion, which does not increase the area of the spacecraft, can maneuver by changing the thrust actively. BETs have also a certain degree of control, because the HC could be electrically connected or disconnected to the bare tether. If disconnected, the tether would be floating and the current almost negligible. The combination of accurate BET simulators, which already exist, with switching on/off the Lorentz force may be used to avoid trackable objects. In the case of DADs, a modification of the frontal area changes the air drag. Sails would need attitude control of a large structure and balloons would need inflation/deflation means. Unlike the simple BET's solution, that is purely electrical, maneuver capability for DADs seems to involve complex space mechanisms.

The discussion of criterion (5), i.e. simplicity and reliability, is not easy for passive technologies because there are not many past missions that could support them. In principle, both technologies would be advantageous because passive physical mechanisms, like air and magnetic drag, are highly preferable as compared with the action-reaction law used by active technologies. The latter normally involve fluids and power on-board, as well as a precise control on the thrust vector or the spacecraft attitude. For electric propulsion this requirement should be accomplished during the long deorbit maneuver. Passive technologies need certain degree of attitude control but only during the short deployment maneuver, which is, probably, the most critical phase of a deorbit mission based on passive technologies. In past suborbital and LEO flights (19 in total), tether deployment was perfect in 14 missions and partial in 4 cases. The safest strategy seems to be a reel system and a cold gas bottle to extract the tether gently. Recent analysis shows that tether cut by small debris is not troublesome if the mission is well designed and tape tether (instead of round) are used [10] (see also Section 4). Besides the deployment of very large structures, DADs have other problems that are not well-resolved or even explored in the past. Many of them are related with the tough space environment that include atomic oxygen attack, ultraviolet radiation and charged particles. Unlike BETs, which involve $\mathrm{Al}$ tapes and mission duration of the order of few months, DADs are normally made of polymers and they need to resist the environment over decades. Possible failures of the sail deployment, like tears in the sail due to entanglement from packaging or polymer degradation after being dormant during the operational life of the spacecraft, are open questions for DADs.

Active technologies, that do not increase the frontal area, satisfy criterion (6). Electrodynamic tethers increase the frontal area but, since $t_{D}$ decreases from decades or centuries to few months, they typically produce a drastic reduction of the product $A \times t_{D}$ with respect to a natural decay. The collision probability between the tether and a spacecraft with representative length $d$, is characterized by the product $t_{D} \times L d$. As shown in Ref. 9, for representative values $d=2 \mathrm{~m}$ and $i=63^{\circ}$, tether missions can be designed with $t_{D} \times L d=203,357$ and $621 \mathrm{~m}^{2}$ year for $m_{s}=37.5,375$ and, $3750 \mathrm{~kg}$, respectively. These values of $A \times t_{D}$ are well below the one found in the literature [14]. In addition, a collision between a BET and an operational spacecraft is not expected to be catastrophic [9]. BET performance would be reduced due to the tether cut but it is highly improbable that the operational spacecraft would be disrupted (see details in Section 4). The probability of a catastrophic collision of the satellite being deorbited with other spacecraft, which is characterized by $t_{D} \times d^{2}$, is obviously even much lower. Regarding DADs, U.S. Government 
Orbital Debris Mitigation standard Practices [15] states that, if drag enhancement devices are to be used to reduce the orbit lifetime, "it should be demonstrated that such devices will significantly reduce the area-time product of the system or will not cause spacecraft or large debris to fragment if a collision occurs while the system is decaying from orbit". As readily follow from the simple model in Section 2, deorbit time is exactly proportional to the DAD's area, thus keeping the $A \times t_{D}$ product unaltered. Certain reduction could be achieved by making the DAD deployment near solar maximum, when the atmospheric density is higher [14]. However, in unfavorable cases, one may wait several years before deploy a drag area.

The only technology that allows a targeted re-entry is chemical propulsion. Therefore, this is the only admissible device for deorbiting large objects that were not Designed for Demise. The most adequate technologies accomplishing criterion (8) are BETs and electric thrusters [16]. BETs would be very efficient because they can be used in thruster and power generation modes [8]. In multi-ADR scenarios, they can store power while deorbiting and use it to feed the BET during the re-boost phase required to approach the different space debris.

\section{Electrodynamic tethers}

Previous discussion suggests that BETs are the most promising devices for deorbiting $\mathrm{S} / \mathrm{C}$ at the end of mission. This section discusses specific requirements of this technology and clarifies certain aspects that have changed in the last years thanks to recent advances. Some of them are results of the FP7/Space project BETs that made important progresses on tether mission design and hardware manufacturing.

Tether dimensioning, in designing for particular operations, brings in the issue of cross-section geometry. Laframboise and Parker proved that the 2D OML current to cylinders of convex cross-section and equal perimeter are equal [17]. This suggested using thin-tape tethers instead of round ones. A fair comparison would consider corresponding tethers (having equal length and mass, and thus equal cross-section area for the same material). This results in equal electric resistance and thus equal maximum (short-circuit limited) current and Lorentz drag. The tape, however, has much greater perimeter and therefore collection capability and faster deorbiting at low plasma density. A tape can reach its maximum current, with current in its corresponding round tether well below maximum. For $w$ (width) $\times h$ (thickness) $=\pi R^{2}$, thin-tape halfperimeter is $w>>\pi R$. Further, tethers being thin and long, they are prone to being cut by small space debris. Since the debris flux decreases rapidly with increasing debris size, the probability of tether cut per unit time is reduced about one to one-and-a-half orders of magnitudes by using tapes [10]. Since they also deorbit faster, tether survivability is greatly increased by using thin tapes.

In principle, multi-line tethers offer the possibility of performing well in both survivability and keeping low mass. Again, for a fair comparison between tapes and multi-line tethers made of multiple wires crossconnected to survive hits by debris, it requires corresponding tethers, which translates into equal cross-section areas,

$f \times N \pi R^{2}=w h$

with the multi-line arrangement made of $N$ round wires and coefficient $f$ $>1$ representing the mass increase over $N$ wires due to cross-connections. The respective half-perimeters are then $N \times \pi R$ and $w$. The condition for the multi-line arrangement having larger perimeter and thus deorbiting faster reads $N>f w / \pi h \sim 500$, for representative values, $w=3 \mathrm{~cm}, h$ $=30 \mu \mathrm{m}$. Note, also, that as $N$ is increased, both the probability of collection interference among the tether lines and the size range of single debris producing cuts increases.

Solid arguments to support the superiority of tapes tether as compared with round tethers in deorbiting missions were given in the $B E T s$ project. A tape tether is much more robust against space debris and, for equal mass and length, it deorbits faster because the collected current is higher due to the larger perimeter [10]. Since the exposition time of the tether to space debris and its geometry is much more favorable for tapes, they exhibit a cut probability by small debris between one and two orders of magnitude smaller than round tethers. This is an important result that may change the awareness raised by past works on tether survivability $[14,18,19]$ in which the investigators considered long $(20 \mathrm{~km})$ and round tethers exposed for years to space debris. It is also remarkable that the 4$\mathrm{km}$ round "fat" tether in TIPs mission survived more than 10 years. It suggests that cut probability by small debris of a tape tether with similar length and flying during few months, which is the relevant case for deorbiting mission, should be very low. Hypervelocity impact tests and numerical simulations conducted at the University of Padova have shown that tape tethers are very resistant to space debris impact in the range below $10 \mathrm{~cm}$ (non catalogued objects), with a maximum number of predicted failures always less than 0.002 over 1 year mission for a $10 \mathrm{~km}$ ( $5 \mathrm{~km} \mathrm{Al}+5 \mathrm{~km}$ inert ) tape tether of width $25.4 \mathrm{~mm}$ [20].

The second cornerstone to carry out fair comparisons of BETs is the correct selection of tether dimensions. Influenced by past tether missions, many of them were designed to smash the world record of the longest man-made object flown in space, many past works assessed tether performance, evaluated mission risk and compared them with other technologies by taking arbitrary dimensions; typically the author considered very long tethers with small and round cross-section. A convenient way to select tether dimensions (length, width and thickness) for given deorbit mission (spacecraft mass and initial orbit altitude and inclination) is the so-called П-algorithm [9]. By combining Eq. (2) and a tether cut probability [10], the time is eliminated to yield the function

$\Pi \equiv \frac{m_{c}}{m_{s}} \times N_{f}=\operatorname{function}\left(w, h, L / h^{2 / 3}, H_{0}, i\right)$

The product of two quantities that should be small for a well-designed mission, i.e. the conductive tether-to-spacecraft mass ratio $\left(\mathrm{m}_{\mathrm{c}} / \mathrm{m}_{\mathrm{S}}\right)$ and the number of fatal impacts by small $(<1 \mathrm{~m})$ debris $\left(N_{f}\right)$, was explicitly written as a function of tether geometry and initial altitude and inclination. The function on the right hand side of Eq. (9) synthetizes information about tether/plasma interaction efficiency, tether design and environmental variables like plasma density, magnetic field and debris flux during the full deorbiting. A first constraint for tether design is given by the minimum exhibited by function $\Pi$ when plotted versus the variable $L / h^{2 / 3}$. A second figure of merit was found by writing Eq. (8) as

$\frac{m_{c}}{m_{s}} \times t_{D}=$ function $\left(L / h^{2 / 3}, H_{0}, i\right)$

Interestingly, this second product does not depend on tether width. These two relations and sensible considerations, like certain upper and lower bounds for tether thickness, lead to optimum missions design.

The П-algorithm is implemented by software BETsMA [21], that plots the two figure of merits given by Eqs.(15) and (16) as a function of tether geometry for a given mission. Once tether geometry is known, BETsMA computes tether deorbiting with more accurate models $[21,22]$. Table 1 shows optimal tether geometry and performance for deorbiting missions from initial orbit altitude $\mathrm{H}_{0}=850 \mathrm{~km}$ and three different inclinations

Table 1

$\mathrm{H}_{0}=850 \mathrm{~km}, \mathrm{H}_{\mathrm{F}}=300 \mathrm{~km}$, epoch 2010, IGRF11, IRI2012, MASTER.

\begin{tabular}{lllllllll}
\hline $\mathrm{i}\left({ }^{\circ}\right)$ & $\mathrm{m}_{\mathrm{s}}(\mathrm{kg})$ & $\mathrm{L}(\mathrm{km})$ & $\mathrm{w}(\mathrm{cm})$ & $\mathrm{h}(\mu \mathrm{m})$ & $\mathrm{m}_{\mathrm{c}} / \mathrm{m}_{\mathrm{s}}(\%)$ & $\mathrm{N}_{\mathrm{f}}(<1 \mathrm{~m})$ & $\mathrm{N}_{\mathrm{f}}(>1 \mathrm{~m})$ & $\mathrm{t}_{\mathrm{D}}$ (days) \\
\hline 25 & 50 & 1 & 1.25 & 10 & 0.7 & 0.006 & 0.0007 & 56 \\
& 500 & 2 & 1.75 & 15 & 0.28 & 0.008 & 0.0023 & 87 \\
& 5000 & 3.75 & 3.25 & 40 & 0.26 & 0.0038 & 0.0047 & 96 \\
63 & 50 & 1.25 & 2.0 & 10 & 1.35 & 0.008 & 0.0017 & 101 \\
& 500 & 3 & 2.75 & 20 & 0.9 & 0.0075 & 0.0041 & 103 \\
& 5000 & 5.5 & 5 & 60 & 0.9 & 0.005 & 0.0084 & 116 \\
98 & 50 & 1.5 & 3 & 12 & 2.9 & 0.0094 & 0.0032 & 164 \\
& 500 & 3.25 & 5 & 20 & 1.7 & 0.0085 & 0.0079 & 185 \\
& 5000 & 7.0 & 6.75 & 80 & 2.0 & 0.001 & 0.0167 & 181 \\
\hline
\end{tabular}


and spacecraft masses. It confirms the first estimations carried out in Section 2 and shows explicitly the good scalability of BETs. Fatal impact rate with small debris, $\mathrm{N}_{\mathrm{f}}(<1 \mathrm{~m})$, was computed by BETsMA. For large debris, $\mathrm{N}_{\mathrm{f}}(>1 \mathrm{~m})$, it used the averaged impact rate $4.80 \times$ $10^{-3} \times L(\mathrm{~km}) \times t_{D}$ (years) [18]. The performance shown in Table 1 should be taken as preliminary. Tether mission design may be the result of an iterative process that will involve detailed simulations including flexible tether dynamics and real hardware manufacturing and testing.

Even in case of collision, it is highly improbable that the tether would disrupt the $\mathrm{S} / \mathrm{C}$ operation. For the most unfavorable case in Table 1, with $h=80 \mu \mathrm{m}$, one finds $\rho_{t} h=216 \mathrm{gr} / \mathrm{m}^{2}$. For a typical length of $d=2 \mathrm{~m}$ characterizing the satellite, the area involved in the collision is $d \times w=$ $0.135 \mathrm{~m}^{2}$ and the impact mass $29.16 \mathrm{gr}$. At a representative relative impact velocity of $10 \mathrm{~km} / \mathrm{s}$, i.e. $5 \times 10^{4} \mathrm{~J} / \mathrm{gr}$, the energy is $1.45 \times 10^{6} \mathrm{~J}$. For this case, $\mathrm{S} / \mathrm{C}$ mass is $5 \mathrm{t}$, thus resulting in a specific impact energy of $0.29 \mathrm{~J} / \mathrm{gr}$ that is well below the threshold required for substantial damage $(10-15 J / g r)$ [23].

A third important progress on tether technology is related with BET dynamics and how the tether attitude instability is avoided by using damping devices that do not require active control. Two damping devices have been proposed and simulated in the BETs study [24]: a rotational damper at the attachment point of the tether to the mother spacecraft and an in-line damper at the attachment point of the tether to the tip mass. Both devices dissipate the kinetic energy passively without any need for sensors and control logic. Simulations indicate that they are capable of keeping the system dynamics stable even in the most demanding cases [25]. For lighter satellites $(\mathrm{m}<200 \mathrm{~kg})$, dynamic stability can be attained with only the in-line damper [26].

The appearance of new materials with extremely low work function $W$ and moderately high temperature stability, like the C12A7: $\mathrm{e}^{-}$electride capable of reaching W below $1 \mathrm{eV}$ [27], could open new opportunities for tether technology. Unlike conventional bare tethers equipped with active plasma contactors, a Thermionic Bare Tether (TBT) has a segment coated with a Low-W material that emits current at moderate temperature, thus bringing the bare tether concept to full completion [28]. Theoretical studies showed the effectiveness of TBT in Geostationary transfer Orbits [29]. Although the absence of HC makes the TBT concept very attractive, a considerable amount of effort has to be dedicated in the future to solve difficulties associated with the thermionic coating and the modelling of the interaction of the TBT with the environmental plasma.

\section{Conclusions}

A comparison of four deorbit technologies according to an especific list of requirements has been done. Simple physical models have been used to estimate the performance of each technology and assess the potential application to deorbit spacecrafts from LEO at the end-of-life. Main drawbacks identified for chemical propulsion, electrical thruster and DAD are mass cost, complexity, and inefficiency at the altitudes of interest, scalability and $A \times t_{D}$ reduction, respectively. Since these problems are linked intimately to the physics of each device, it is not expected that technology development will overcome them in the future. The physics of BETs, i.e. Lorentz drag, seems to be more favorable than action-reaction law and air drag in deorbiting scenarios, because it is passive and effective at the altitudes of interest. However, two aspects should be demonstrated for BETs. The first one is the manufacturing of a deorbit kit based on BETs where its full mass $k_{B E T} m_{c}$ has $k_{\mathrm{BET}}$ below about four, as here assumed. This is a technological challenge that mainly requires the integration in a single kit of a deployment mechanism with a passive damper, a HC system, a tape tether and a power control module. Previous works in the FP7/Space project BETs suggest that this goal is possible. The recent appearance of the TBT concept, which is free of HC and consumables, can play an important role to make the system even simpler. However, a considerable amount of research has to be done in areas like plasma physics and material science to make the TBT concept feasible. The second cornerstone is a demonstration flight of a bare tether with hollow-cathode that would help to dissipate doubts related with mission risks.

A fair comparison shows that BETs may dominate other technology in terms of performance and reliability. The word fair is used here to highlight the importance of recent BET progress. A fair comparison should consider a tape tether, and its geometry has to be selected according to the initial orbit and the S/C mass. Our estimations show that tape tethers have much better performance in deorbiting missions in LEO than round, multi-line tethers and other tether concept like e-sails, which also requires a considerable amount of power on-board. The simulations indicate that a well-designed tape tether with lengths equal to few $\mathrm{km}$ can deorbit S/C in the ton range within few months and with a low risk.

\section{Acknowledgments}

Work by G. S-A is supported by the Ministerio de Economía y Competitividad of Spain under Ramón y Cajal Programme (Grant No. RYC-2014-15357).

\section{References}

[1] D.J. Kessler, B.G. Cour-Palais, Collision frequency of artificial satellites: the creation of a debris Belt, J. Geophys. Res. 83 (No. A7) (1978) 2637-2646 (1 Jun.).

[2] C. Bonnal, Design an operational practices for the passivation of spacecraft and launchers at the end of life, J. Aerosp. Eng. 221 (Part G) (2007) 925-931.

[3] C. Bonnal, Space Debris mitigation measures applied to European launchers, Acta Astronaut. 65 (2009) 1679-1688.

[4] J.C. Liou, A. Rossi, H. Krag, M.X.J. Raj, K. Anilkumar, T. Hanada, H. Lewis, Stability of the future LEO Environment, IADC 12-08, Rev. 1 (2013).

[5] J. Sanmartín, Propellantless deorbiting of space debris by bare electrodynamic Tethers, 51th Session of the Scientific Subcommittee, United Nations Committee on the Peaceful Uses of Outer Space, 10-21 February, Viena, 2014.

[6] SPADES CDF Study Report, SPADES, assessment of solid propellant autonomous deorbit System, April, CDF Study Report: CDF-137(A), 2013.

[7] L. Herbeck, C. Sickinger, M. Eiden, M. Leipold, Solar sail hardware development, European Conference on Spacecraft Structures, Materials and Mechanical Testing, Toulouse, 11-13 December, 2002.

[8] J. Sanmartín, M. Martinez-Sánchez, E. Ahedo, Bare Wire anodes for electrodynamic tethers, J. Propuls. Power 9 (3) (1993) 353-360 (May-June).

[9] J. Sanmartín, A. Sánchez-Torres, B.S. Khan, G. Sánchez-Arriaga, M. Charro, Optimum sizing of bare-tethers for deorbiting satellite at end of mission, Adv. Space Res. 56 (7) (2015) 1485-1492 (October).

[10] S.B. Khan, J.,R. Sanmartín, Survival Probability of round and tape tethers against debri impact, J. Spacecr. Rockets 50 (3) (2013) 603-608.

[11] J.R. Sanmartin, E.C. Lorenzini, M. Martinez-Sanchez, Electrodynamic tether applications and constraints, J. Spacecr. Rockets 47 (2010) 3 (May-June).

[12] P. Janhunen, Electrostatic plasma brake for deorbiting a satellite, J. Propuls. Power 26 (2) (2010) 370-372.

[13] J. Sanmartín, R.B. Estes, The Orbital-motion-limited regime of cylindrical langmuir robes, Phys. Plasmas 6 (1) (1999) 395-405.

[14] K.T. Nock, K.M. Aaron, D. McKnight, Removing orbital debris with less Risk, J. Spacecr. Rockets 50 (2) (2013) 365-379.

[15] U.S. Government, Orbital Debris Mitigation Standard Practices, NASA/http:// orbitaldebris.jsc.nasa-gov/library/USG_OD_standard_practices.pdf $\rangle, 2000$.

[16] L. Savioli, A. Francesconi, F. Maggi, L. Olivieri, E. Lorenzini, C. Pardini, Space debris removal using multi-mission modular spacecraft, 6th European Conference on Space Debris, ESA/ESOC, Darmstadt, Germany, 22-25 April, 2013, L. Ouwehand (ed), 6b.O-11

[17] J.G. Laframboise, L.W. Parker, Probe design for orbit-limited current collection, Phys. Fluids 16 (1973) 629-636.

[18] L. Anselmo, C. Pardini, The survivability of space tether system in orbit around the Earth, Acta Astronaut. 56 (2005) 391-396.

[19] W.J. Cooke, D.B. Spencer, B.J. Anderson, R.M. Suggs, Tether survivability and collision avoidance: is LEO the right place for tethered systems? 2001 Space Technology and Applications International Forums (STAIF-2001),Institute for Space and Nuclear Power Studies, University of New Mexico (Feb.) 2001.

[20] A. Francesconi, C. Giacomuzzo, F. Branz, E.C. Lorenzini, Survivability to Hypervelocity impacts of electrodynamic tape tethers for deorbiting spacecraft in LEO. in: Proceedings of the Sixth European Conference on Space Debris, ESA/ESOC Darmstadt, Germany, 22-25 April, 2013.

[21] G. Sánchez-Arriaga, C. Bombardelli, X. Chen, The impact of nonideal effects on bare electrodynamic tether performance, J. Propuls. Power 31 (3) (2015) 951-955.

[22] M. Sanjurjo-Rivo, G. Sánchez-Arriaga, J. Pelaez, Efficient computation of current collection in bare electrodynamic tethers in and beyond OML regime, J. Aerosp. Eng. 28 (6) (2015) 04014144.

[23] D. McKnight, R. Maher, L. Nagl, Refined Algorithms for structural breakup due to hypervelocity impact, Int. J. Impact Eng. 17 (1995) 547-558. 
[24] R. Mantellato, M. Pertile, G. Colombatti, A. Valmorbida, E.C. Lorenzini, Deorbiting of spacecraft at the end of life with electrodynamic tethers stabilized by passive oscillation dampers, in: CEAS Space Journal, 6, Elsevier, 2014, pp. 133-143.

[25] BETs Team, Orbital simulation report, Technical Report D14.2 prepared by University of Padova-CISAS for European Commission project 262972 (BETs), FP7 SPA.2010.2.3-2, March, 2014.

[26] R. Mantellato, A. Valmorbida, M. Pertile, A. Francesconi, E.C. Lorenzini, G. Sánchez-Arriaga, End-of-life deorbiting services for microsatellites making use of bare electrodynamic Tethers. Proceedings of Small Satellites Systems and Services Symposium (The 4-S Symposium), 26-30 MayPorto Petro, Majorca, Spain, 2014.
[27] Y. Toda, S. Matsuishi, S. Hayashi, K. Ueda, T. Kamiya, M. Hirano, H. Hosono, The impact of field emission of electron anions clathrated in subnanometer-sized cages in $\left[\mathrm{Ca}_{24} \mathrm{Al}_{28} \mathrm{O}_{64}\right]^{4+}(4 \mathrm{e}-)$,, Adv. Mater. 16 (8) (2004) 685-689.

[28] J.D. Williams, J.R. Sanmartín, L.P. Rand, Low work-function coating for an entirely propellantless bare electrodynamic tether, IEEE Trans. Plasma Sci. 40 (5) (2012) 1441-1445.

[29] G. Sanchez-Arriaga, X. Chen, E. Lorenzini, Optimal design and deorbiting performance of thermionic bare tethers in geostationary transfer orbits (http:// dx.doi.org/), J. Propuls. Power, Accept. Online (2016), http://dx.doi.org/10.2514/ 1.B36202. 